\title{
Integration of Geomagnetic Disturbance Modeling into the Power Flow: A Methodology for Large-Scale System Studies
}

\author{
Thomas J. Overbye, Trevor R. Hutchins, Komal Shetye \\ University of Illinois at Urbana-Champaign \\ Urbana, IL, USA \\ overbye@illinois.edu, hutchns2@illinois.edu, \\ shetye1@illinois.edu
}

\author{
Jamie Weber, Scott Dahman \\ PowerWorld Corporation \\ Champaign, IL, USA \\ weber@powerworld.com, scott@powerworld.com
}

\begin{abstract}
This paper presents a methodology for integrated power flow modeling of the impact of geomagnetic disturbances (GMDs) on the power system voltage stability. GMDs cause quasi-dc, geomagnetically induced currents (GICs) in the transformers and transmission lines, which in turn cause saturation of the high voltage transformers, greatly increasing their reactive power consumption. GICs can be calculated using standard power flow modeling parameters such as line resistance, augmented with several GIC specific fields including substation geographic coordinates and grounding resistance, transformer configuration, and transformer coil winding resistances. When exact values are not available estimated quantities can be used. By then integrating GIC into power flow analysis, the changes in reactive power losses and bus voltages can be quantified to assess the risk of voltage instability and large-scale voltage collapse. An example calculation is provided for a North American Eastern Interconnect model.
\end{abstract}

\section{I.INTRODUCTION}

The potential for a geomagnetic disturbance (GMD) to severely impact the operation of electric power systems worldwide is an area of growing concern. An example of this is the February 2012 release of a special reliability assessment on GMDs by NERC [1], which notes that there are two risks that occur with the introduction of geomagnetically induced currents (GICs). The first is the potential for damage transmission system assets, primarily the high voltage transformers. The second is the loss of reactive power support leading to the potential for a voltage collapse. The focus of this paper is on the second risk, considering the power flow modeling needed to provide an assessment of GIC related voltage stability risks with a particular emphasis on the practical aspects of doing such calculations for large-scale systems.

\section{OVERVIEW OF GIC POWER FLOW MODELING}

The basic modeling methodologies associated with the representation of GICs in the power flow have been well

This work was partially funded by EPRI and by the US Department of Energy through "The Future Grid to Enable Sustainable Energy Systems: An Initiative of the Power Systems Engineering Research Center (PSERC) described in the literature. As noted in [2], it has been known since at least the early 1940's that GMDs have the potential to impact the power grid. This is due to GMD-related changes in the earth's magnetic field inducing quasi-dc electric fields in the earth (with frequencies usually much below $1 \mathrm{~Hz}$ ) with the electric field's magnitude and direction GMD event dependent. These electric fields in-turn cause GICs in the high voltage grid. These quasi-dc currents can then cause halfcycle saturation in the power transformers, resulting in increased transformer reactive power losses. The reactive power losses are usually assumed to varying linearly with the GICs in the transformer [2], [3].

The inclusion of the impact of GICs in the power flow was first described in [4]. The gist is that the GICs can be determined by first solving a dc network of the form

$$
\mathbf{I}=\mathbf{G} \mathbf{V}
$$

in which $\mathbf{G}$ a square matrix similar in form to the power system bus admittance matrix, except 1) it is a real matrix with just conductance values, 2) the conductance values are determined by the parallel combination of the three individual phases, 3) $\mathbf{G}$ is augmented to include the substation neutral buses and substation grounding resistance values, and 4) the transformers are modeled with their winding resistance to the substation neutral and in the case of autotransformers both the series and common windings are represented. The voltage vector $\mathbf{V}$ contains entries for the substation neutral dc voltages and the bus de voltages.

Two main methods have been proposed for modeling the impact of the GMD-induced electric field variation in the power grid: either as dc voltage sources in the ground or as dc voltage sources in series with the transmission lines [4], [5]. In both approaches the dc voltages are represented as Norton Equivalent currents in the I vector of (1). In [5] it was shown that while the two methods are equivalent for uniform electric fields, only the transmission line approach can handle the nonuniform electric fields that would be expected in a real GMD event. Therefore here the impact of the magnetic field 
variation is represented by dc voltage sources in series with each of the transmission lines.

Using the approach of [5], to calculate the GMD-induced line voltage the electric field is just integrated over the length of the transmission line. In the common approach of assuming a constant electric field over the length of the transmission line (recommended in [1] for planning studies), the dc line voltage can be calculated as

$$
V=E_{N} L_{N}+E_{E} L_{E}
$$

where $E_{N}$ is the Northward electric field $(\mathrm{V} / \mathrm{km}), E_{E}$ is the Eastward electric field $(\mathrm{V} / \mathrm{km}), L_{N}$ is the Northward distance $(\mathrm{km})$, and $L_{E}$ is the Eastward distance $(\mathrm{km})$. With a uniform field (2) is independent of the actual path of the transmission line; just knowing the geographic location of the line's terminal buses is sufficient. In the case of non-uniform fields the voltage can be approximated by dividing the line into segments, applying this procedure to the individual segments, and then summing the results. While such an approach would be path dependent, because GMDs are continental in scope, the small variation in the electric field over most line lengths would not be significant. Therefore (2) provides an adequate approximation. A twenty bus test system demonstrating these calculations is provided in [6].

How the GICs flow in the electric transmission system depends upon the induced dc voltage in the transmission lines, the resistance of the various system elements, and the available paths to ground. Since the GICs are essentially dc, device reactance plays no role in their determination other than recognizing that at dc frequencies capacitors look like an open circuit. Hence shunt capacitors are ignored, and transmission line series capacitors block GICs on their lines. Values that impact the GICs include the resistance of the transmission lines, the resistance of the coils of grounded transformers, the resistance of the series windings of autotransformers (and their common winding if grounded), and the substation grounding resistance.

This is illustrated for a simple two generator, four bus network in Figure 1 with Bus 1 and its generator (Bus 3) in Substation A, and Bus 2 with its generator (Bus 4) in Substation B. Assume Buses 1 and 2 are joined by a $765 \mathrm{kV}$ line that has a per phase resistance of $3 \Omega$, the per phase resistance of the high side (grounded side) coil of each of the two transformers is $0.3 \Omega$, and the grounding resistance for each of the substations is $0.2 \Omega$. Since the concept of per unit plays no role in GIC determination, resistance values are expressed in Ohms $(\Omega)$, current is in amps (A), and the dc voltages are given in volts $(\mathrm{V})$. Assume the substations are at the same latitude, separated by $150 \mathrm{~km}$ in the east-west direction, with an assumed electric field of $1 \mathrm{~V} / \mathrm{km}$ in the eastwest direction. This gives an induced voltage in the transmission line of $150 \mathrm{~V}$.

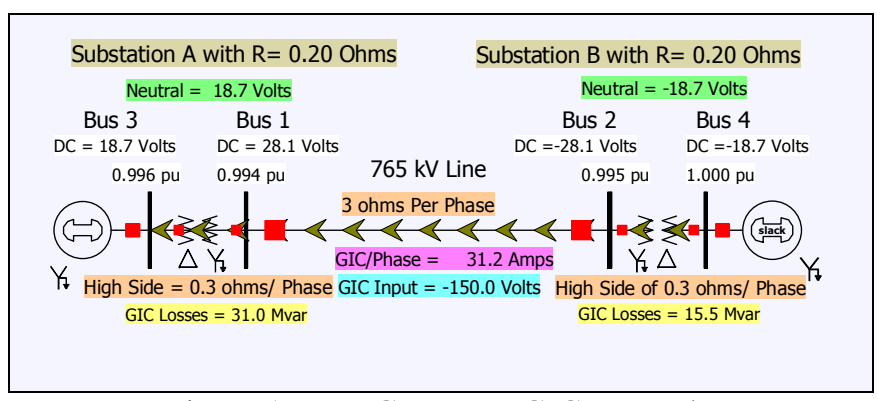

Figure 1: Two Generator GIC Example

The GICs can then be determined by solving a simple dc circuit. From a GIC perspective the three phases for the transmission line and transformers are in parallel, so the total three phase resistance for the $765 \mathrm{kV}$ line is $(3 / 3) \Omega=1 \Omega$, and $(0.3 / 3) \Omega=0.1 \Omega$ for each of the transformers. These resistance are then in series with the Substation A and B grounding resistance giving

$$
I_{\text {GIC }, 3 \text { Phase }}=\frac{150 \text { volts }}{(1+0.1+0.1+0.2+0.2) \Omega}=93.75 \mathrm{amps}
$$

with the flow from ground into the high side coil of the Substation B transformer, down the $765 \mathrm{kV}$ line, into the high voltage coil in Substation A and back into the ground. In the figure the direction and size of the brown arrows are used to visualize the direction and magnitude of the GIC flow.

The substation neutral and bus dc voltages can then be calculated by a straightforward application of Ohm's law. For example, the Substation B neutral voltage is $(0.2 \Omega) *(-93.75 \mathrm{~A})$ $=-18.75 \mathrm{~V}$, while the Bus 2 voltage is $(-18.75 \mathrm{~V})+(0.1 \Omega) *(-$ $93.75 \mathrm{~A})=-28.1 \mathrm{~V}$. Because of the delta connection on the low side of the transformers, no GIC passes through the transformers. Since the generators are assumed to be grounded through a low resistance into the substation neutral, their dc bus voltage is the same as their substation neutral.

A potential point of confusion in interpreting the results of the GIC calculations is to differentiate between the per GIC phase currents in transmission lines and transformers, and the total GIC three phase current in these devices. From a results display perspective either could be shown. Since the three phases are in parallel, the conversion between the two is straightforward with the total current just three times the per phase current. The convention commonly used for GIC analysis is to use the per phase current for transformers and transmission lines. Thus the GIC in the example is $93.75 / 3=$ 31.25 A/per phase.

Once the GICs have been calculated, the next step in the analysis is to determine the GIC-related transformer reactive power losses. As mentioned earlier a linear function can be used [2], [3] with [7] making the observation that these reactive power losses vary linearly with terminal voltage. Therefore for each transformer the losses could be written as

$$
Q_{\text {Loss }}=V_{k V} k I_{G I C}
$$

where $\mathrm{Q}_{\mathrm{Loss}}$ is the transformer's GIC-related reactive power loss in Mvar, $\mathrm{V}_{\mathrm{kV}}$ is the terminal voltage (in $\mathrm{kV}$ ), $\mathrm{I}_{\mathrm{GIC}}$ is the 
per phase GIC in the transformer (in amps) and $\mathrm{k}$ is a transformer specific constant.

However, since the power flow expresses voltages in per unit (pu), the approach used here is to embed the transformer's maximum nominal voltage in the constant rewriting (4) as

$$
Q_{\text {Loss }}=V_{p u} K I_{G I C}
$$

where $\mathrm{V}_{\mathrm{pu}}$ is the pu voltage and $\mathrm{K}$ has units of Mvars/amp.

This approach works fine when $\mathrm{K}$ is available for each individual transformer. In large system studies, in which default values for $\mathrm{K}$ are often used, an alternative approach is to modify (5) slightly to use an assumed nominal voltage in the definition of $\mathrm{K}$. Then the constant value needs to be scaled based upon the transformer's actual maximum nominal $\mathrm{kV}$ level. The reactive power loss equation then becomes

$$
Q_{\text {Loss }}=V_{p u} K\left(\frac{V_{\text {Nom kV }}}{V_{\text {Nom kV,Assumed }}}\right) I_{G I C}
$$

where $\mathrm{V}_{\mathrm{Nom} \mathrm{kV}}$ is the nominal $\mathrm{kV}$ of the highest winding for the transformer, and $\mathrm{V}_{\mathrm{Nom}} \mathrm{kV}$,Assumed is the assumed nominal voltage. If $\mathrm{K}$ is specified for a particular transformer then the assumed value is just equal to the nominal and (6) is identical to (5). When used as a default for a number of transformers with varying nominal values the value used here is $500 \mathrm{kV}$.

The value of $\mathrm{I}_{\mathrm{GIC}}$ used in (6) is an "effective" value that depends on the type of transformer. In the simplest case of a grounded wye-delta, such as is common for generator step-up transformers (GSUs), $\mathrm{I}_{\mathrm{GIC}}$ is straightforward - just the current in the grounded coil. For transformers with multiple grounded windings and autotransformers the value of $\mathrm{I}_{\mathrm{GIC}}$ depends upon the current in both coils [4]. Here we use the approach of [8], which shows that the equations for $\mathrm{I}_{\mathrm{GIC}}$ for an autotransformer can be generalized as

$$
I_{G I C}=\left|\frac{a_{t} I_{H}+I_{L}}{a_{t}}\right|
$$

where $I_{H}$ is the per phase dc current going into either the high side winding or the series winding of an autotransformer, $\mathrm{I}_{\mathrm{L}}$ is the per phase dc current going into either the low side winding or the common winding of the autotransformer, and $a_{t}$ is the transformer turns ratio defined as

$$
a_{t}=\frac{N_{H}}{N_{L}}=\frac{N_{S}+N_{C}}{N_{C}}
$$

Completing the example, since both of the GSUs are grounded only on the high side, $\mathrm{I}_{\mathrm{GIC}}=\mathrm{I}_{\mathrm{H}}=31.25 \mathrm{~A}$ for both. The increased transformer reactive power is calculated using an assumed $\mathrm{K}$ of 1.0 Mvar/A for the left GSU, and 0.5 Mvar/A for the right GSU. Because of the linear dependence of reactive power on bus voltage magnitude, in the power flow it is represented as a constant reactive current.

\section{LARGE-SCALE ISSUES ASSOCIATED WITH THE} DETERMINATION OF THE GICS

This section considers the practical aspects of associated with GIC determination for a large system, showing that these calculations can be effectively integrated into existing power flow packages. An example is provided using a 62,000 bus model of the North America Eastern Interconnect (EI) with the solution done using PowerWorld Simulator with the GIC add-on [9]. With respect of prior work in the large system area, very few papers have addressed these practical considerations. Such large-scale studies have certainly been done, with [10] providing results both from a simulation of the 1989 GMD that impacted the North American Eastern Interconnect (including blacking out the Hydro Quebec system), along with an assessment of the potential for future GMDs to affect the U.S. power grids. While [10] does provide a wealth of useful information about such studies, it does not provide a detailed solution methodology.

From a conceptual point of view, determining the GICs in a large system is very similar to the methodology introduced with the four bus example. That is, knowledge of a GMD storm scenario and an appropriate power system model allows one to determine the current vector and conductance matrix in (1). This equation is then solved to determine the voltage vector. From a computational perspective this solution is almost trivial, taking less than one second for the 62,000 bus model considered (significantly less than the associated power flow solution). The voltage vector is then used to determine the $\mathrm{I}_{\mathrm{GIC}}$ for all of the system transformers, then (6) is used to determine the increased transformer reactive power demand.

All of these steps just involve the solution of linear equations so they are fast and reliable. For some GIC studies just calculating these values is sufficient. However, if desired, the power flow equations could also be solved with the increased reactive power loading at each transformer modeled as a reactive current load.

Much of the data needed for GIC analysis is contained in the standard power flow models (for example the PSSE Raw File Format [11]). This includes the network topology, bus voltage levels, resistance of the transmission lines and the presence of transmission line series compensation.

For transformers, the power flow model contains the total series resistance of the transformer but does not contain the resistance of the individual windings. When available the actual winding resistance should be used. Otherwise the individual coil winding resistances can be estimated by recognizing that the total resistance is not equally split between the two windings. Rather, since the high voltage winding has more turns and lower amps, its resistance will be higher. Referring to (8), a ballpark ratio of the high to low winding resistance is $\left(a_{t}\right)^{2}$ for a regular transformer and $\left(a_{t}-1\right)^{2}$ for an autotransformer. Thus for a non-autotransformer the winding resistances can be estimated using

$$
\frac{R_{p u}}{R_{\text {Base, HighSide }}}=R_{\text {HighSide }}+a_{t}^{2} R_{\text {LowSide }}
$$


and assuming the magnitude of both terms on the right-hand side of (9) are equal. For an autotransformer the equation is

$$
\frac{R_{p u}}{R_{\text {Base,HighSide }}}=R_{\text {Series }}+\left(a_{t}-1\right)^{2} R_{\text {Common }}
$$

Transformer winding configurations (e.g., wye or delta) and grounding are not usually included in the power flow model, but they can either be determined from short circuit data or estimated. The estimated values are grounded wyegrounded wye for transmission level transformers and deltagrounded-wye for transmission to distribution transformers (with the delta on the transmission side to keep zero sequence distribution current out of the transmission system). Resistance values are not needed for the ungrounded windings. Hence when a load is modeled at the transmission level it is modeled as having no ground connection because of the implicit delta winding on the transmission side.

For generators the modeling trend is to explicitly represent the GSU in the power flow model. If the GSU is not modeled (i.e., the generator is directly connected to its high voltage bus), the resistance of the GSU is sometimes included in auxiliary power flow fields (e.g., the generator RT field from [11]). Usually for a GSU the vast majority of the resistance is on the high voltage side. Since the common GSU configuration is grounded wye on the high side, and delta on the low (generator) side, the low side winding and the generator can be ignored.

One key data structure needed for GIC analysis is substation records. While some power flow packages have long contained explicit substation records, others do not. Substation records are needed to 1) modeling the grounding resistance required for the construction of $\mathbf{G}$ in (1), 2) represent the substation neutral voltages and current injections in the $\mathbf{V}$ and $\mathbf{I}$ vectors of (1), and 3) provide the geographic locations needed for the calculation of dc line voltages in (2). In the analysis presented here (using [9] which does contain substation records) the power flow data was read in from a Raw File [11], which did not contain substation data. Then an auxiliary file was used to create the substations with their associated latitude and longitude and map the buses into the substations. The 62,000 bus EI example considered here has more than 27,000 substations.

The substation grounding resistance field is used to represent the effective grounding resistance of the substation, consisting of its grounding mat and the ground paths emanating out from the substation such as due to shield wires grounding [8]. This resistance depends upon several factors including the size of the substation (with larger substations generally having a lower value) and the resistivity of the ground (with substations in rocky locations having higher values). Ballpark values for low resistivity soil are usually substantially below $0.5 \Omega$ for a $230 \mathrm{kV}$ and above substations, and between 1 and $2 \Omega$ for the lower voltage substations. Figure 2 plots the sorted assumed substation grounding resistances used in this example. While the figure contains some actual data, most of it is estimated, with high voltage substations and those with more incident lines (and presumably a larger grounding mat) having lower values.

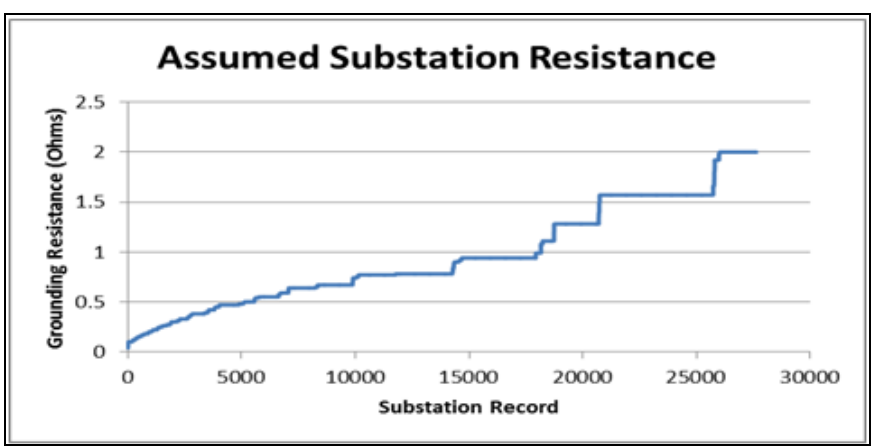

Figure 2: Sorted Substation Grounding Resistance

The final piece needed to calculate the system GICs is an assumed GMD scenario. As noted in [1, pp. 64], if the intent of a study is to reproduce an actual storm then detailed models are needed that include the geology of the study area; if the purpose of the study is for planning purposes then a constant electric field can be assumed with different studies looking at different electric field magnitudes and directions. Since this paper focuses on planning applications, just uniform fields are used. Also, because (1) and (2) are linear, the GICs for all field magnitudes and directions can be determined with superposition by just solving for the east-west and for the north-south field directions for a specified field value.

As an example, Figure 3 and Figure 4 visualization the GICs calculated in the North American EI case for (respectively) an east-west field and a north-south field of the same field strength, just using data contained in the power flow model, along with estimated defaults for substation grounding resistance and reasonably accurate bus geographic locations. The yellow arrows show the direction and magnitude of the GICs. The purpose for these figures is not to advocate that a uniform field across an entire interconnect would actually occur. Rather it is to demonstrate that these values can be quickly determined and visualized, with the calculations need to taking on the order of one second.

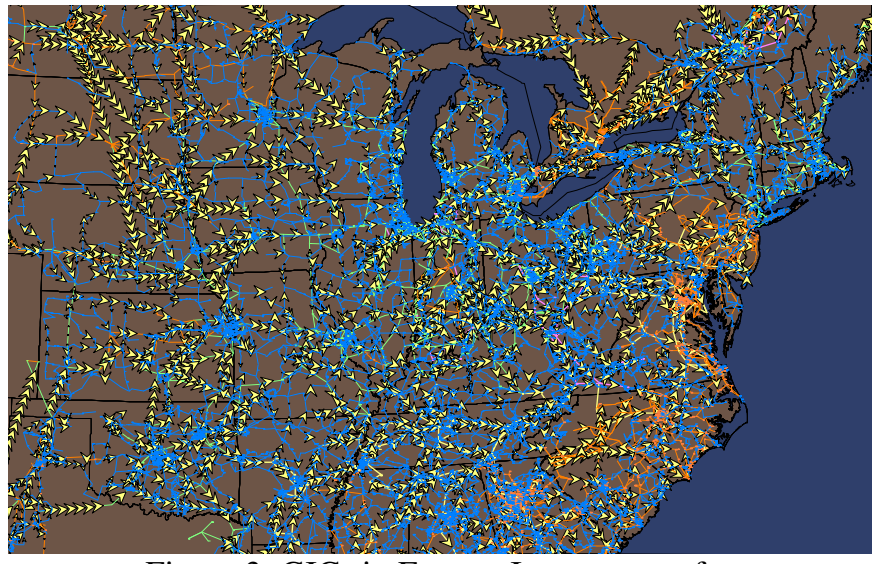

Figure 3: GICs in Eastern Interconnect for Uniform East-West Field 


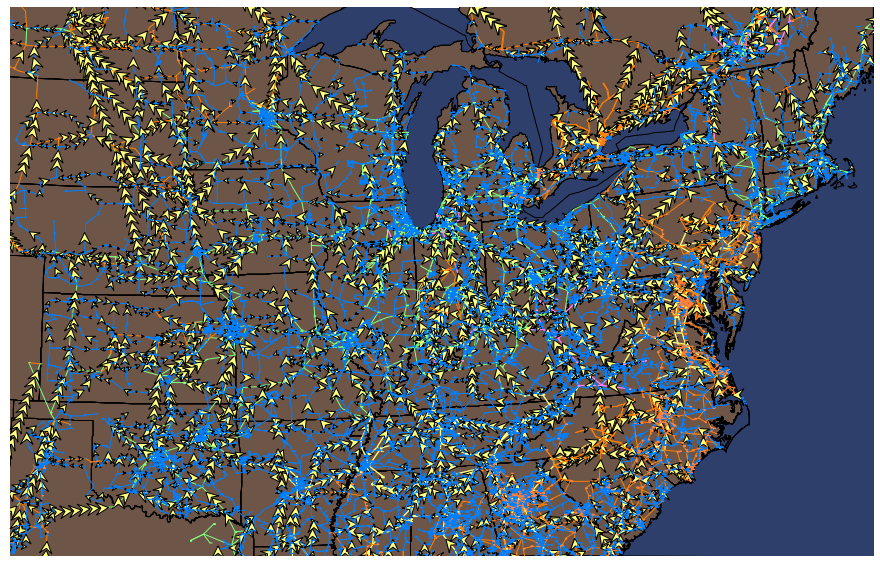

Figure 4: GICs in Eastern Interconnect for Uniform North-South Field

An important issue then in performing a GIC study for a particular geographic footprint (e.g., a utility or balancing authority area) is determining what electric field values to use in areas outside of the footprint. The analytic dependence of the GIC flow on a particular line or transformer going between buses $\mathrm{j}$ and $\mathrm{k}$ or transformer to another line's GIC-induced voltage (going between buses $\mathrm{m}$ and $\mathrm{n}$ ) can be easily determined by solving

$$
\Delta \mathbf{V}=[\mathbf{G}]^{-1} \Delta \mathbf{I}
$$

in which the only non-zeros in $\Delta \mathbf{I}$ would be equal and opposite values at the positions for buses $m$ and $n$, and the only desired values in $\Delta \mathbf{V}$ are at the positions for buses $\mathrm{j}$ and $\mathrm{k}$. Sparse vector methods could be used for fast computation of such sensitivities [12].

Pragmatically the result is that the GMD induced voltages only tend to affect electrically nearby buses. As an example Figure 5 shows a zoomed view of Figure 4 except that GMD voltages are only assumed on transmission lines in the TVA service territory (footprint) ${ }^{1}$. Now the resultant GICs are almost exclusively contained on the TVA lines. As a specific example, a $500 \mathrm{kV}$ line in the middle of TVA has a Figure 5 (TVA only) current equal to $99.7 \%$ of its Figure 4 (full system) value while a $345 \mathrm{kV}$ transmission line in Central Illinois has a TVA only value of essentially zero (less than $0.1 \%$ of its full system value). Conversely, if the voltages are assumed to be zero only in TVA the first line's GIC is now $2.8 \%$ of its full system value, and the Illinois line is at $99.99 \%$. Thus when performing a GIC study electric field values are only important for the footprint of interest and nearby buses. So little error is introduced in the GICs themselves if one considers outside lines to have no electric field or the same electric field as the footprint of interest.

\footnotetext{
${ }^{1}$ An online map of the Tennessee Valley Authority (TVA) area is available at http://www.tva.gov/sites/sites_ie.htm
}

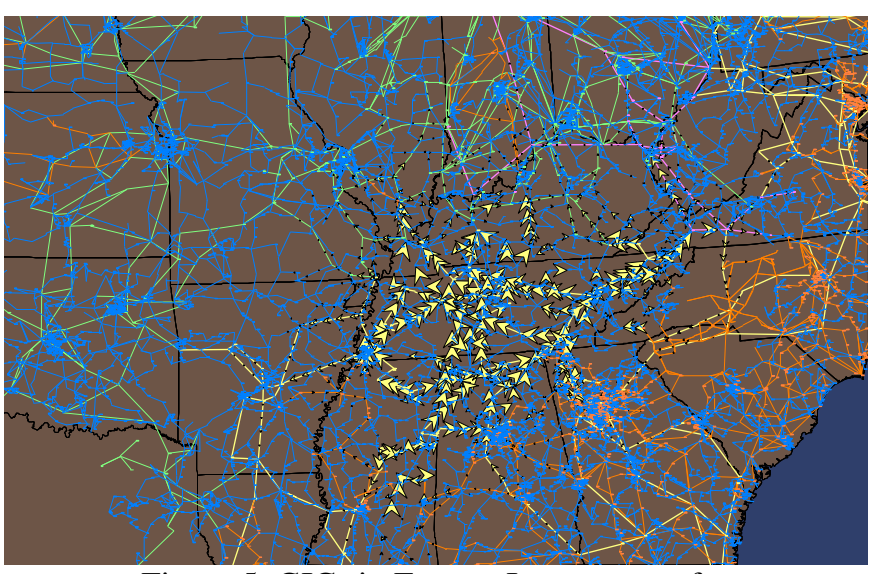

Figure 5: GICs in Eastern Interconnect for Uniform North-South Field

Another issue to address is whether the lower voltage transmission grid needs to be included in the GIC calculations. The NERC General Simulation Guidelines say that transmission lines below $230 \mathrm{kV}$ are typically not modeled due to the higher resistance values of the lower voltage lines [1, pp. 64]. In testing this guideline using the EI case the GIC associated Mvar losses with the transmission lines below 230 $\mathrm{kV}$ ignored in (1) were $87 \%$ of their value full case values for an east-west field and $85 \%$ for a north-south field. However, these values differed substantially for the individual areas in the case. In the Ontario the guideline appears to be justified since the total Mvar value with the lines below $230 \mathrm{kV}$ ignored was $99 \%$ of when they were included. But in other areas, such as New England and Michigan, the reactive loss value with the lines ignored was less than $60 \%$ of when they were included. Therefore is general guideline may need to be reconsidered, but it may be justified for certain locations. Also, given the low computational costs there are few reasons to exclude the lower voltage lines.

\section{LARGE-SCALE ISSUES ASSOCIATED WITH GICS INTEGRATED INTO THE POWER FLOW}

Once the GICs have been determined (6) is used to determine the increased reactive power losses for each transformer. These losses are then modeled in the power flow as a constant reactive current. While conceptually these additional reactive power losses could be calculated by an external program and then imported into the power flow, the more convenient approach presented here is to include the GIC calculations integrated into the power flow solution.

Regardless of approach, the impact the GICs have on the power flow, and whether they would ultimately cause a voltage collapse is dependent upon the assumed $\mathrm{K}$ parameter from (6). In general $\mathrm{K}$ is transformer dependent with its exact value either determined from detailed study of the transformer's structure or through measurement. Measurement opportunities only occur during GMD events, but with increased transformer dc neutral current monitoring hopefully better known of these values will be forthcoming.

Nevertheless, the literature does provide some guidance on its selection. In [3] the following transformer values are

Submitted to 2012 North America Power Symposium (NAPS), September 2012, Champaign, IL 
presented based upon the transformer's core design: $K=1.18$ for a single phase core, 0.33 for a shell form, 0.29 for a 3legged and 0.66 for a 5-legged core; but [3] does not mention the assumed voltage level. In [10, pp. 1-21] a $\mathrm{K}$ value of about 1.7 (normalized to $500 \mathrm{kV}$ ) is presented for single phase transformers, while in [13] a value of 1.1 (again normalized to $500 \mathrm{kV}$ ) is presented for these transformers, and about 0.8 for a shell form and slightly less for a 5-legged core. Since single phase transformers are usually only used for transformers at or above $500 \mathrm{kV}$, a worst case analysis might be to use a $\mathrm{K}$ of say 1.7 for $500 \mathrm{kV}$, and a value of about 0.8 (normalized to $500 \mathrm{kV}$ ) for all others. These assumptions were used in the results presented here. Obviously if the exact $\mathrm{K}$ value is known for a transformer then it should be used.

The last issue to consider in doing GIC power flow study is the size of the study area. Since the GICs increase transformer reactive power consumption, the concern is whether the system will have sufficient reactive reserves to avoid voltage collapse. Due to the relatively high reactances of the transmission lines, it is widely recognized that reactive power does not travel far in the transmission grid, so voltage stability concerns are local or at most regional [14].

Hence the GIC power flow study approach presented here is to assume a uniform electric field over the entire case, but to only calculate the GICs in the areas of interest (AOI), and nearby buffer areas. The GIC induced transformer reactive losses are then also modeled only at the transformers in these areas. The electric field is then increased until the power flow no longer converges (i.e., close to the voltage collapse point).

In the specific example presented here the AOI consisted of 1781 buses, with buffer areas of 3264 buses (out of a case total of 62605 buses). The case represented 2012 anticipated summer peak conditions. An east-west field was assumed, which caused the total transformer GIC related losses to increase at a rate of about 716 Mvars per $1 \mathrm{~V} / \mathrm{km}$ increase in the assumed field (a value that dropped slightly as the bus voltages decreased). The initial reactive power generation in the AOI and buffer areas was 10,400 Mvar (out of a case total 128,646 Mvar), with the lowest initial transmission level voltage (above $100 \mathrm{kV}$ nominal) in these areas at $0.934 \mathrm{pu}$.

The electric field was then increased in $0.5 \mathrm{~V} / \mathrm{km}$ increments, with the last valid power flow solution occurring with an assumed field of $11.5 \mathrm{~V} / \mathrm{km}(18.5 \mathrm{~V} / \mathrm{mile})$. For reference the GMD that blacked-out Quebec in 1989 had peak electric fields of $2 \mathrm{~V} / \mathrm{km}$ [15], while according to [16] a 100 year storm could cause peak fields of $20 \mathrm{~V} / \mathrm{km}$ in Quebec (a "resistive" region) and $5 \mathrm{~V} / \mathrm{km}$ in British Columbia (a "conductive" region). With an assumed field of $11.5 \mathrm{~V} / \mathrm{km}$ the total reactive generation in the AOL and buffer areas had increased by about $67 \%$ to 17,419 Mvars. The lowest transmission level voltage was $0.866 \mathrm{pu}$, with sixty transmission buses having voltages below $0.9 \mathrm{pu}$. The highest transformer effective $\mathrm{I}_{\mathrm{GIC}}$ was $334 \mathrm{~A} /$ phase, with 33 transformers having IGIC values above $100 \mathrm{~A} / \mathrm{phase}$. The impact of these currents on transformer heating and loss of life was not considered in this study.

\section{SUMMARY AND FUTURE DIRECTIONS}

The paper has presented a methodology for including GMD assessment as an integrated part of the power flow solution, with results presented for a large-scale system. The paper shows that with standard power flow data, reasonable assumptions for other GIC parameters and an assumed uniform electric field, the solution is relatively straightforward. The necessary study tools now exist for integrating GMD assessment into the power system planning process.

There are many directions for future work. Certainly a key issue is validation, which will be greatly facilitated by the growing number of direct GIC measurements in the power system. Another area of research is the development of algorithms for determining optimal GIC mitigation strategies, which could include both operational strategies when a GMD is imminent and longer-term solutions such as the installation of GIC reduction devices. Improved geographical modeling is needed to determine the validity of the constant electric field and the appropriate field values to use in particular geographic areas. Finally, more specific system studies are needed to determine whether significant damage to system assets such as transformers would occur before a voltage collapse due to lack of reactive support.

\section{REFERENCES}

[1] "2012 Special Reliability Assessment Interim Report: Effects of Geomagnetic Disturbances on the Bulk Power System," NERC, Feb. 2012.

[2] V.D. Albertson, J.M. Thorson Jr., R.E. Clayton, S.C. Tripathy, "Solar-Induced-Currents in Power Systems: Cause and Effects," IEEE Trans. on Power Apparatus and Systems, vol.PAS-92, no.2, pp. 471-477, March/April 1973.

[3] X. Dong, Y. Liu, J.G. Kappenman, "Comparative Analysis of Exciting Current Harmonics and Reactive Power Consumption from GIC Saturated Transformers," Proc. IEEE 2001 Winter Meeting, Columbus, OH, Jan. 2001, pp. 318322.

[4] V.D. Albertson, J.G. Kappenman, N. Mohan, and G.A. Skarbakka, "Load-Flow Studies in the Presence of Geomagnetically-Induced Currents," IEEE Trans. on Power Apparatus and Systems, vol. PAS-100, Feb. 1981, pp. 594606.

[5] D.H. Boteler, R.J. Pirjola, "Modeling Geomagnetically Induced Currents Produced by Realistic and Uniform Electric Fields," Proc. IEEE Trans. on Power Delivery, vol. 13, Oct. 1998, pp. 1303-1308.

[6] R. Horton, D.H. Boteler, T.J. Overbye, R.J. Pirjola, R. Dugan, "A Test Case for the Calculation of Geomagnetically Induced Currents," Submitted to IEEE Transactions on Power Systems, March 2012.

[7] R.A. Walling, A.H. Khan, "Characteristics of Transformer Exciting Current During Geomagnetic Disturbances", IEEE Transactions on Power Delivery, Vol. 6, No. 4, October 1991 
[8] K. Zheng, et. al., "Influence of System Characteristics on the Amplitudes of Geomagnetically Induced Currents," Submitted to IEEE Trans on Power Delivery, March 2012.

[9] PowerWorld Simulator GIC Analysis, http://www.powerworld.com/products/GIC.asp.

[10] J. Kappenman, "Geomagnetic Storms and Their Impacts on the U.S. Power Grid," Metatech Corporation Report MetaR-319, Jan. 2010.

[11] PSSE Raw File Format, https://www.ptius.com/PTI/software/psse/raw_data_format.cfm.

[12] W.F. Tinney, V. Brandwajn, S.M. Chan, "Sparse Vector Methods," IEEE Trans. on Power Apparatus and Systems, vol. PAS-104, February 1985, pp. 295-301.
[13] R. Walling, "Transformer Response to GIC Flow," EPRI/NERC GIC Modeling Workshop, Atlanta, GA, April 2012.

[14] R.A. Schlueter, "A Voltage Stability Security Assessment Method," IEEE Trans. on Power Systems, vol. 13, Nov. 1998, pp. 1423-1438.

[15] D. Boteler, "Geomagnetically Induced Currents: Present Knowledge and Future Research," IEEE Trans. on Power Delivery, vol. 9, Jan. 1994, pp. 50-58.

[16] A. Pulkkinen, E. Bernabeu, J. Eichner, C. Beggan, A. Thomson, "Generation of 100-year Geomagnetically Induced Current Scenarios," Space Weather, April 2012. 